\title{
Biomass accumulation in a biofilter treating toluene at high loads. Part 2: Model development, calibration and validation
}

\author{
Antonio David Dorado $^{\mathrm{a} *}$, Javier Lafuente ${ }^{\mathrm{b}}{\text {, David } \text { Gabriel }^{\mathrm{b}} \text { and Xavier Gamisans }}^{\mathrm{a}}$ \\ ${ }^{a}$ Department of Mining Engineering and Natural Resources, Universitat Politècnica de Catalunya, Bases de \\ Manresa 61-73, 08240 Manresa, Spain \\ ${ }^{\mathrm{b}}$ Department of Chemical Engineering, Universitat Autònoma de Barcelona, Edifici C, 08193 Bellaterra, \\ Barcelona, Spain \\ *Corresponding author e-mail: dorado@emrn.upc.edu; Tel: +34 9387772 36; Fax: +34 938777202
}

\begin{abstract}
In this work, a dynamic model describing volatile organic compounds abatement and the corresponding biomass accumulation is developed, calibrated and validated. The mathematical model is based on detailed mass balances which include the main processes involved in the system: advection, absorption, adsorption, diffusion, biodegradation and biomass growth. The model overcomes common assumptions considered in classical biofiltration models such as uniform, constant biomass distribution. The model was calibrated and validated using experimental data obtained from a biofilter packed with clay pellets during its operation from inoculation to clogging. The model was able to predict satisfactorily experimental data by calibrating only a minimum number of parameters such as the halfsaturation constant for toluene and the volumetric maximum growth rate of microorganisms. Kinetic parameters were fitted by means of an optimization routine using toluene concentration profiles along the bed height of the biofilter. A confidence interval for each parameter was calculated based on the Fisher Information Matrix procedure. The model was satisfactorily validated during the operation of the biofilter under different process conditions. Biomass accumulation permitted to predict macroscopic, critical operating parameters such as the pressure drop through the bed. The model may help predicting energy consumption requirements as well as biomass clogging episodes due to excessive biomass growth.
\end{abstract}

Keywords: biomass growth, kinetic parameters estimation, modeling, toluene abatement, high loads, biofiltration. 


\section{INTRODUCTION}

Although biological gas treatment has become an effective and economical alternative to traditional systems, biofilter modeling has been scarcely developed due to the complexity of describing the fundamental processes and due to the lack of reliable physical, chemical and biological parameters. Although more than 200 experimental works focusing on VOCs biofiltration have been published in the last 5 years, less than 40 studies dealing with mathematical modeling of toluene removal by biofiltration can be found in literature in the same period of time. Amongst them, simple and complex models have been employed to model biofiltration under both steady-state and dynamic operating conditions [1-6]. Dynamic models are generally more interesting since they could serve to predict biofilters performance and for process control and optimization [7]. However, most dynamic models in biofiltration rarely incorporate biomass accumulation processes, even if experimental observations do not correspond with this common assumption. Instead, a constant and uniform biomass distribution is commonly assumed. Then, important scenarios in full-scale bioreactors operation such as bioreactors startup, starvation periods and clogging episodes are not wellpredicted if biomass accumulation processes are not considered in the model. In addition, since biomass density is strictly related with the availability and concentration of pollutant in the gas-phase, predicted results could differ significantly from experimental data in the case of calibration at low loads and operation at high pollutant loads.

Several authors have addressed biomass growth and modeling from different approaches. Arcangeli and Arvin [8] modeled the biodegradation of toluene and the associated biofilm growth in a biodrum system (a cylinder rotating inside another with the biomass growing on the surface of the rotator and the stator). Alonso et al. [9] developed a model considering a homogeneous biomass for one type of microbial species attached to uniformly shaped solids expressing mass balance equations in spherical coordinates. However, parameters related with biomass growth, e.g. pressure drop, were not described by model predictions. Iliuta et al. [10] modeled toluene abatement to illustrate the consequences of excessive biomass formation. Kinetics for cell growth and substrate degradation were evaluated from a suspended cell culture and, then, assumed equal to those of the immobilized system. Recently, VergaraFernandez et al. [11] focused on biological treatment of hydrophobic compounds by a specific fungi consortium. Their model describes the increase in the transport area by the growth of 
the filamentous mycelia and the relation with n-hexane abatement. Alvarez-Hornos et al. [12] studied the modeling of a mixture of toluene and ethyl acetate in view of cross-inhibition between substrates. However, biofilm growth with time was assumed negligible although stratification of the biomass density in the axial direction was taken into account by means of an empirical expression [13].

According to the stated above, the aim of this work is to contribute to the general understanding on how biomass growth can be modeled in a biofilter by means of simple expressions. A general dynamic biofiltration model for toluene as model pollutant describing biomass growth is developed, calibrated and validated. Mathematical equations are based on discretized mass balances taking into account the main chemical, physical and biological processes involved in the system. Previous experimental results obtained by Dorado et al. [14], in which high loads of toluene were treated by biofiltration from inoculation to clogging, were used in the present work as input data to calibrate and validate the model. A rigorous statistical test is used in order to assess the accuracy of model predictions. The model is aimed at predicting observable process variables such as biomass weight, pressure drop across the biofilter and toluene concentrations.

\section{MODEL DEVELOPMENT}

The model developed in the present study was built based on the previous model of Dorado et al. [15], which considers the most relevant phenomena occurring during the biofiltration process like advection, absorption, adsorption, diffusion and biodegradation. In the present model, biomass growth and its effect on those operational parameters commonly monitored in biofiltration (specially pressure drop) is included. Dynamic mass balances in the gas phase and within the biofilm including biomass growth serve to describe changes in the biodegradation capacity of the biofilter during operation, overcoming the limitations of most biofiltration models which assume a constant biomass concentration in space and/or in time.

The reactor was divided into 3 different phases in which mass balances are applied: 1) a gas phase, where the pollutant is transported by advection; 2) a biofilm phase, where the pollutant is transferred from the gas phase and is biologically degraded, and 3) a solid phase made of packing material where biofilm grows and pollutant can be adsorbed. As biomass grows, the 
biofilm thickness increases and fills the void volume of the bed at expenses of the gas phase, thus reducing the empty bed section for the air circulation. Therefore, bed porosity decreases along the biofilter operation, causing an increase in the system pressure drop.

Important basic assumptions underlying the model are based on consolidated models reported in literature $[5,6,15]$. Specific assumptions were added to account with biomass growth modeling:

(1) Biofilm is formed on the surface of the packing material. Model considers that biomass grows on the surface of the packing material covering all the pores, discounting micropores $\left(\mathrm{a}_{\mathrm{M}}\right)$, and forming a sphere whose area (a) is considered available for transferring the pollutant from the gas phase. Thus, biomass grows in the external surface and also in the internal macro pores of the packing material but only the external surface is available to transfer pollutant from the gas phase. Reactions only take place in the biofilm phase.

(2) Biofilm thickness is uniform at the same height of the biofilter. Thus, radial biomass distribution is not considered. In the experimental setup, air inlet was distributed uniformly at the bottom of the biofilter and the ratio between particle size and reactor diameter was superior to 1:30 to avoid wall effects [16].

(3) Adsorption of pollutant onto the support is described by the corresponding isotherm obtained in a previous characterization of the packing material [17].

(4) Biomass detachment is described by a biomass decay coefficient $\left(\mathrm{k}_{\mathrm{d}}\right)$ based on the amount of biomass collected in the leachate as a consequence of wash out during watering periods.

(5) The entire material surface is covered by the biofilm even though the thickness depends on axial position and time.

\subsection{Mass balance and kinetic expression}

Mass balances in the gas and biofilm phases, kinetics and rate equations for the substrate (C) and biomass $(\mathrm{X})$ as well as algebraic equations for time-dependent parameters defined in the present work are shown in Table 1. Unlike previous mathematical models, where bed porosity $(\varepsilon)$, interstitial velocity $\left(v_{z}\right)$, biofilm thickness $(\delta)$ and biomass concentration $(X)$ are often considered constant, in the model presented herein all parameters are described in dynamic 
conditions as a function of the change in biomass density due to biomass growth during reactor operation. This corresponds to a more realistic description of the phenomena occurring in a biofilter.

In the gas phase, pollutant circulates by advection and is transferred to the biofilm according to Eq.1. The interstitial gas velocity is calculated as a function of the porosity of the reactor bed (Eq. 2) while the mass flux is given by Fick's law (Eq. 3). In the biofilm phase, the pollutant diffuses and is biologically degraded (Eq.4). Because of the high pollutant loads tested and the sorption capacity of the packing material used, a sorption isotherm determined in wet conditions of the packing material (20) was considered at $\mathrm{x}=\delta$. Biomass growth rate is based on the net change of biomass density according to Eq. 5 .

Although several kinetic expressions have been used to describe VOCs biodegradation such as zero or first-order kinetics $[4,18]$, most of recent works relate the specific consumption rate for toluene degradation through a Monod-type equation as this work considers (Eq. 6). In the case of toluene abatement, although some authors indicate that at loads higher than $1000 \mathrm{~g} \cdot \mathrm{m}^{-}$ ${ }^{3} h^{-1}$ toluene becomes inhibitory for bacterial activity [19], inhibition effects have not been detected in the experimental period of the present study [14].

The pollutant degradation rate depends on the biomass growth rate and a biomass to substrate yield coefficient $\left(\mathrm{Y}_{\mathrm{p}}\right)$ (Eq. 7). The relation $\mathrm{V}_{\mathrm{R}} / \mathrm{V}_{\mathrm{b}}$ is needed in this expression because biomass concentration $\mathrm{X}$ is expressed as $\mathrm{g}$ biomass per $\mathrm{m}^{-3}$ reactor. To be consistent with the mass balance in the biofilm phase this parameter is referred to the biofilm proportion in the total volume of the reactor. In this sense $C_{b}$ is the concentration of pollutant referred to the biofilm volume. The biofilm volume (Eq. 8) and the biofilm thickness (Eq. 9) increase or decrease proportionally to the biomass concentration over the packing material. Accordingly, the bed porosity is reduced as a consequence of an increased biofilm thickness as expressed in Eq. 10. Either provided by the packing material or externally fed through the watering system, nutrient concentration was considered to be constant and not limiting along the height of the reactor during biofilter operation.

Pressure drop was predicted according to the equation defined in Dorado et al. [17]. A modified Ergun equation was adjusted for several packing materials taking into account the 
dynamic hold-up, the bed porosity and the gas flow rate. In the work herein, only the bed porosity was a time-dependent variable in the modified Ergun equation.

\subsection{Model calibration and validation}

The set of partial differential equations was discretized in space along the bed height and biofilm thickness. The conversion of the tubular reactor into a serial of stirred reactors was verified running simulations at different discretizations and optimizing results and time computing. As a result, an optimal discretization of the biofilter was found, resulting in eight nodes along the bed height and eight nodes along the biofilm thickness.

The resulting set of ordinary differential equations was solved using MATLAB in a homemade modeling environment. A variable order method was used for solving stiff differential equations based on the numerical differentiation formulas (NDFs), which are generally more efficient than the closely related family of backward differentiation formulas (BDFs), also known as Gear's methods. The maximum time step used in the numerical solution routine was established in 1 day in accordance with the time interval of the experimental data. Since the inlet toluene concentration and inlet gas flow changed throughout biofilter operation, a linear interpolation was considered for the time interval comprised between two consecutive inlet data. All inlet concentrations and air flowrates collected in the experimental procedure were used as input variable of the model. If the data requested by the computing system is at a different time than those registered during the experiment, the model interpolates between the two closest times.

The calibration procedure was mainly based on direct experimental determination as well as on curve fitting of experimental data monitored during biofilter operation. The volumetric maximum growth rate $\left(\mu_{\max }\right)$ and the half-saturation constant $\left(\mathrm{K}_{\mathrm{s}}\right)$ were the set of parameters estimated by curve fitting during the model calibration step. All the other parameters of the model, except for the diffusion coefficient and the Henry constant for toluene that were obtained from the literature [2], were experimentally determined.

To start with the calibration procedure, initial guesses were assigned to kinetic parameters according to the literature. Predicted outlet concentrations by the model were compared with the experimentally measured data and the deviations between both were used to obtain 
updates for kinetic parameters. Parameter values were sought to minimize the objective function $(O F)$ given in Eq. (11).

$$
O F=\sqrt{\sum_{i=1}^{N} \sum_{j=1}^{M}\left[C_{g, j}\left(\mu_{\max }, K_{s}\right)-C_{g, j}^{*}\right]^{2}}
$$

where $\mathrm{M}$ is the total number of sampling ports along the biofilter height (4 in the present study B,C,D,E); $C_{g, j}$ is the concentration of toluene in gas phase predicted by the model in $\mathrm{g}$ $\mathrm{m}^{-3}$ in the sampling port $\mathrm{j} ; \mu_{\max }$ and $K_{S}$ are the unknown kinetic parameters to fit; $C_{g, j}^{*}$ is the concentration of toluene experimentally measured in gas phase in $\mathrm{g} \mathrm{m}^{-3}$ at the sampling port $\mathrm{j}$; and $\mathrm{N}$ is the number of measurements realized along the operation time. The total number of data sets are $\mathrm{M} \cdot \mathrm{N}$ and equal to 52 comparisons.

The parameter estimation was performed using a MATLAB algorithm based on a multidimensional unconstrained nonlinear minimization (Nelder-Mead) algorithm. This is a direct search method that does not use numerical or analytical gradients. Model calibration and validation of the present work was checked by performing a statistic analysis based on a paired t-Student's test at 5\% level of significance.

Calibration and validation of the dynamic model was carried out using part of the experimental data of the previous work of Dorado et al. [14], in which the biofilter performance was studied when treating high loads of toluene under different operating conditions in a period of 120 days. In the work presented herein, the first 600 hours of operation after the start up were chosen for calibrating the model. The experimental period was selected within the interval time where operating conditions were more controlled and uniform. Thus, a pseudo steady-state operation was experimentally verified. In this period, the biofilter packed with clay pellets was operated at an average inlet load of $600 \mathrm{~g}$ toluene $\mathrm{m}^{-3} \mathrm{~h}^{-1}$ at an EBRT of 35s. The pollutant load treated in this period was high enough to detect a concentration profile along the height of the biofilter. Subsequently, the inlet toluene load was stopped and the performance of the biofilter was observed under starvation conditions. In this period, an important part of accumulated biomass was washed due to watering. After 20 days without inlet feed, the biofilter was resumed to a different pollutant load $\left(390 \mathrm{~g} \cdot \mathrm{m}^{-3} \cdot \mathrm{h}^{-1}\right)$. The resumption period was used to validate the model with the kinetic parameters calibrated in the 
previous period. Toluene abatement, pressure drop and biofilter weight, among others, were monitored to study the suitability of the dynamic model.

\section{RESULTS AND DISCUSSION}

\subsection{Model calibration}

Model calibration was based on the determination of the volumetric maximum growth rate and the half-saturation constant. Model parameters used in the present work are shown in Table 2. The initial bed porosity (0.38) was experimentally determined in Dorado et al. [14]. The biofilm density was calculated by direct measurement of volume and weight of the immobilized biomass extracting part of the packing material at frequent operation times and scraping biomass from the surface. The initial biomass concentration and the biomass-tosubtrate yield was determined in Dorado et al. [14] by correlating the toluene load and the $\mathrm{CO}_{2}$ production. According to equation (6), the decay coefficient was obtained from the slope of the log evolution of the total solid collected in the leachate It was assumed that dead biomass was completely detached and washed out by the periodic irrigation.

A volumetric maximum growth rate of $0.20 \pm 0.01 \mathrm{~h}^{-1}$ and half-saturation constant of $0.0060 \pm 3 \cdot 10^{-4} \mathrm{~g}$ toluene $\mathrm{m}^{-3}$ were found for the steady operation period of the biofilter. Confidence intervals were estimated according to the Fisher Information Matrix (FIM). The FIM matrix summarizes the quantity and quality of information obtained during the experimental procedure. In the FIM estimation the output sensitivity functions of the model, the number of experimental data and the errors of experimental measurements are considered $[15]$.

Alonso et al. [9] reported a volumetric maximum growth rate of $0.125 \mathrm{~h}^{-1}$ and a halfsaturation constant of $0.15 \mathrm{~g}$ toluene $\cdot \mathrm{m}^{-3}$ in a biotrickling filter packed with a randomly pelletized medium operating at an EBRT between 1 and 2 min. Similarly, Arcangeli and Arvin [8] reported a value of $0.15 \mathrm{~h}^{-1}$ and $0.6 \mathrm{~g}$.toluene $\mathrm{m}^{-3}$ for both kinetic parameters, respectively. Dorado et al. [15] found a volumetric maximum growth rate of $1.3 \mathrm{~h}^{-1}$ and a half-saturation constant of $0.16 \mathrm{~g}$ toluene $\mathrm{m}^{-3}$ for a bacterial community in a biofilter packed with coconut fiber and pine leaves. It is important to highlight that none of them modeled so high inlet toluene loads as in the present work, which had an impact on the kinetic parameters 
estimated. While the maximum volumetric rate is almost similar to previous works, the halfsaturation constant is around three orders of magnitude lower than previously reported values, which may indicate that the affinity between toluene and the microbial population developed in the present biofilter is enhanced at these operational conditions.

Toluene concentrations predicted by the model after curve fitting of the kinetic parameters $\left(\mu_{\max }\right.$ and $\mathrm{K}_{\mathrm{S}}$ ) describe satisfactorily the evolution of experimental data in the 4 sampling ports distributed along the height of the biofilter (Figure 1). The statistical analysis based on a paired t-student test performed underlines the good correspondence between model predictions and experimental data at 3 different operation periods: at the start of the monitoring period (day 6), in the middle of the operation time (day 9) and at the end of the period (day 24). Despite the good correlation between model and experimental data in the biofilter profiles, concentrations predicted by the model deviate slightly away in the last days of the calibration period $(\mathrm{t}=580 \mathrm{~h})$. Observed differences may be partially explained by processes not considered in the description of the model such as, irregular biomass growth due to the creation of preferential flow channels or the proliferation of dead zones. These processes are more important at the end of the calibration period when the amount of biomass accumulated over the packing material is higher. Subsequently, Figure 2 shows the toluene concentration profiles at the different sampling ports predicted by the dynamic model in comparison to all concentrations measured experimentally. Predictions obtained in the first module (B), in which higher gas-phase concentrations were found, showed the best fitting among all experimental data.

Biomass production predicted by the model corresponded well with those parameters measured experimentally to monitor biomass growth. Pressure drop monitored during the operation shows a good agreement with results simulated by the model despite of the high fluctuations in the measurement of this parameter (Figure 3a). Likewise, the decrease in bed porosity due to the biomass growth is well-described by the assumptions and mathematical expressions of the model (Figure 3b). Additionally, biomass weight increase due to biomass accumulation measured in the lab-scale plant was also verified by model predictions (Figure 3c). According to the description of the processes involved in the biological system, the expected biomass production also agrees with observed results. At the end of the period, biomass weight predicted is slightly underestimated, which is explained by the more significant water retention achieved due to the decrease in bed porosity and the difficulties of 
trickling water from irrigation. Overall, it could be affirmed that biomass growth was satisfactorily described by the model.

\subsection{Model validation}

The dynamic model was validated in the same lab-scale plant than in the calibration step but under different operating conditions [14]. While kinetic parameters were calibrated for the first steady-state operation period, model validation was performed by comparing the simulation results to experimental data in a posterior time lapse of operation (days 75 to 95). In between both periods, the biofilter was starved for 20 days and continuously watered (300 $\left.\mathrm{mL} \cdot \mathrm{d}^{-1}\right)$ in order to wash biomass accumulated in the packed bed.

Model predictions for the resumption period are shown in Figure 4, illustrating an excellent agreement with experimental data. Additionally, a statistical analysis based on a paired tstudent test was performed in the validation process in order to quantify the agreement between results predicted by the model with the optimized kinetic parameters and experimental data. The t-test executed on the outlet toluene concentration measured at the different sampling ports yielded an absolute value of 0.83 for the validation period. A t-value of 2.01 at a $5 \%$ level of significance for 54 degrees of freedom indicates that the difference between toluene concentration measured experimentally and toluene concentration predicted by the model are not statistically significant in the studied period [22]. Moreover, both the progressive increase in the toluene concentration fed to the biofilter and the load shut-down at the end of the simulated period are perfectly described by the model in the outlet and intermediate ports during the simulation period. As in the calibration step, bed porosity and biomass weight (data not shown) as well as the evolution of pressure drop (Figure 5) are in good agreement with experimental data.

Therefore, taking all the results into consideration, it can be stated that the proposed model describes satisfactory biomass accumulation and the rest of phenomena occurring in the biofiltration of a common volatile organic compound (toluene). From a practical point of view, model predictions of pressure drop may be very useful to assess a wide range of operating conditions and to carefully estimate the operating cost related with air blowing through the reactor and, thus, prevent bed clogging. Including biomass accumulation in the mathematical model may be useful in order not to underestimate the energy consumption 
requirements of a plant and to obtain a more accurately estimation for calculating the financial cost of blowing air through a biofilter.

\subsection{Model interpretation}

In order to interpret model results, Figure 6 shows the biofilm thickness growth during the biofilter startup for the 4 sampling ports (Figure 6A) and thickness profiles along the height at different times after startup (Figure 6B). As expected, biomass growth is more significant at the lower part of the biofilter where pollutant concentration is higher. This difference becomes more important along the time. Biomass growth starts being perceptible in the first module after $72 \mathrm{~h}$ of operation while around $225 \mathrm{~h}$ are needed to detect a significant biofilm development in the last module of the biofilter. The biomass distribution profile is flat during the first days and becomes sharper after $300 \mathrm{~h}$ of operation. As pollutant concentration and biofilm thickness are higher at the inlet of the biofilter, the maximum degradation rate is always obtained close to this region, which is perfectly consistent with experimental results.

The dynamic behavior of the model is explained by the following observation. Initially, the biomass concentration inside the biofilter is extremely low so that the removal efficiency (RE) is practically zero. As sorption capacity of packing material is relatively low at wet conditions, the material is saturated in few minutes at the high inlet concentrations. As time goes by, with a minimum biomass density grown on the support, pollutant load is practically abated, showing a minimum outlet toluene concentration. In this time span, toluene abatement is limited by biodegradation due to a low microorganism concentration. For the upper modules, as the gas-phase concentration is low, the biofilm phase concentration becomes also inferior. Consequently, the degradation rate is lower and the outlet toluene concentration increases until a quasi-steady value fixed by the biodegradation capacity of microorganisms and the mass transfer processes involved in the process.

It is noteworthy that the present model considers neither a uniform biomass nor a constant distribution within the biofilter. The biomass distribution in time is only consequence of microorganisms' growth which depends on pollutant availability and bio-kinetic parameters. Although uniform biomass is a common assumption used in biofiltration modeling, the model predictions for the abatement of high loads of pollutant may differ significantly from the observed real behavior. Moreover, clogging is one of the most important operation problems related with long term biofilter operation which could be satisfactory prevented by model 
predictions taking biomass growth into consideration in the description of biofilters behavior. For instance, in the present work model could predict, before starting the real operation, that operating at these conditions for a biofilter of the same characteristics, the porosity will decrease a $60 \%$ in 25 days. Predicting biomass clogging may be very useful to program maintenance tasks and to estimate packing material replacement or cleaning and their economical connotations. In this way, operational conditions can be optimized to favor longterm operations at lower cost.

A sensitivity analysis was performed with the mathematical model to identify those parameters with a higher influence in model predictions. Sensitivity analysis was performed for each parameter, including all kinetic, stoichiometric and physicochemical parameters. This part of the study was assessed by increasing and decreasing by $10 \%$ the values of the parameters and comparing the relative change of the state variables (outlet pollutant concentration and elimination capacity) to a relative change of the value of the parameter. Results revealed that some essential parameters should be carefully determined to minimize deviations from real behavior modeled. Specifically, operational parameters (inlet concentrations, flow rate and reactor dimensions) firstly, and specific surface area and partition coefficient secondly, are the most sensitive among the rest. Immediately afterwards, kinetic parameters show strong influence on final results. On the contrary, initial bed porosity and initial biomass thickness expose low dependence which it is important to underline since both parameters are comparably more difficult to experimentally determined. Interestingly, looking at previous sensitivity analysis performed in the former model by Dorado et al. [15], present results indicate that the most sensitive parameters in the ranking remain almost unaltered after adding the biomass growth equations and corresponding parameters.

\section{CONCLUSIONS}

A dynamic model to simulate toluene abatement and biomass accumulation has been developed, calibrated and validated through a set of different experimental conditions for a biofilter treating toluene at high loads. The rigorous model was able to predict satisfactorily experimental data by calibrating only a few numbers of parameters and a relatively simple system of equations. Estimated parameters not only predict the removal capacity of the biofilter under different operating conditions, but also describe satisfactorily those parameters 
affected by biomass growth under dynamic conditions. Pressure drop, bed reactor porosity and biomass weight were properly described by model predictions. Therefore, the model can be useful in the prevention of clogging by excessive growth of biomass and in the estimation of operating costs in a wide range of conditions. Model validation was verified using a rigorous statistical test which corroborates the accuracy of model estimation in several operating environment.

\section{ACKNOWLEDGMENTS}

Antonio David Dorado received a pre-doctoral scholarship of the MEC (Ministerio de Educacion y Ciencia, Spain). Authors acknowledge the financial support provided by the “Comision Interministerial de Ciencia y Tecnologia”, (CICYT) and the European Regional Development Fund (ERDF-EC), projects CTQ 2006-14997-C02-02 and CTQ 2009-14338C03-03. The Department of Chemical Engineering at UAB is a unit of Biochemical Engineering of the Xarxa de Referencia en Biotecnologia de Catalunya (XRB), Generalitat de Catalunya.

\section{SYMBOLS USDED}

a specific surface area of transfer (surface area per unit volume of bed reactor) in $\mathrm{m}^{2} \mathrm{~m}^{-3}$

$a_{m} \quad$ specific surface area of material (surface area per unit volume of bed reactor) in $\mathrm{m}^{2} \mathrm{~m}^{-3}$

A cross-section area of the bioreactor in $\mathrm{m}^{2}$

$C_{b} \quad$ pollutant concentration in the biofilm phase in $\mathrm{g} \mathrm{m}^{-3}$

$C_{g} \quad$ concentration of toluene in the gas phase in $\mathrm{g} \mathrm{m}^{-3}$

$C_{g i} \quad$ inlet gas concentration in $\mathrm{g} \mathrm{m}^{-3}$

$C_{g j} \quad$ concentration of toluene in gas phase predicted by the model in $\mathrm{g} \mathrm{m}^{-3}$ in $\mathrm{j}$

$C_{g j}^{*} \quad$ concentration of toluene experimentally measured in gas phase in $\mathrm{g} \mathrm{m}^{-3}$ in $\mathrm{j}$

$C_{s} \quad$ pollutant concentration in the support material in $\mathrm{g} \mathrm{m}^{-3}$

$D \quad$ diffusion coefficient in $\mathrm{m}^{2} \mathrm{~h}^{-1}$

$H \quad$ gas-liquid distribution coefficient given by Henry’s law

$j \quad$ index of the sampling port

$k_{d} \quad$ detachment coefficient in $\mathrm{h}^{-1}$

$K_{S} \quad$ semi-saturation or affinity constant in $\mathrm{g} \mathrm{m}^{-3}$

$N \quad$ number of measurements realized along the operation time 


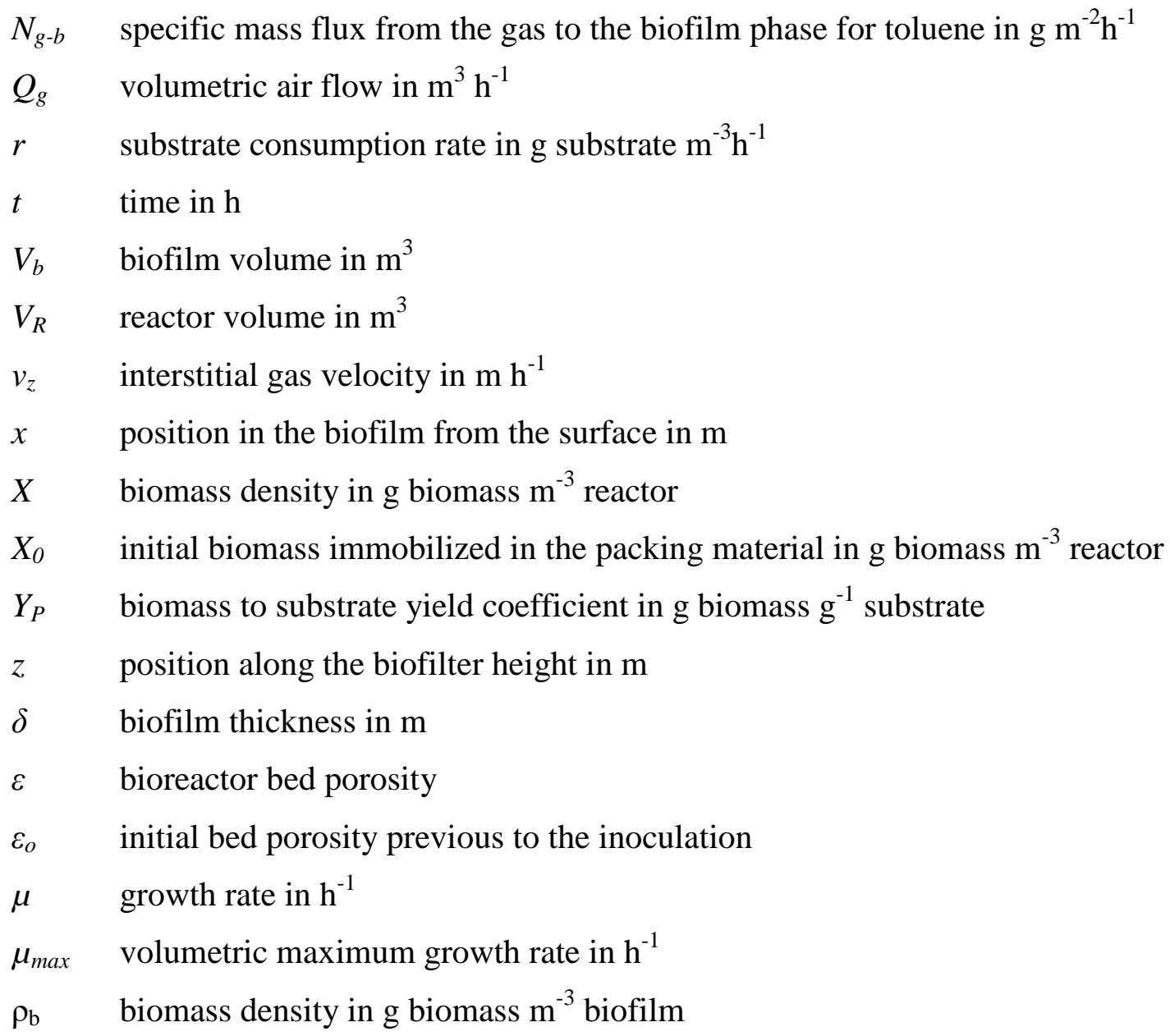

\section{REFERENCES}

[1] R.J. Abumaizar, E.H. Smith, W. Kocher, Analytical model of dual-media biofilter for removal of organic air pollutants, J. Environ. Eng. 123 (1997) 606-614.

[2] M. Amanullah, S. Farooq, S. Viswanathan, S, Modeling and simulation of a biofilter. Ind. Eng. Chem. Res. 38 (1999) 2765-2774.

[3] A. Metris, A. M. Gerrard, R. H. Cumming, P. Weigner, J. Paca, Modelling shock loadings and starvation in the biofiltration of toluene and xylene, J. Chem. Technol. Biotechnol. 76 (2001) 565-572.

[4] S. Ottengraf, H.C. Van der Oever, Kinetics of organic compunds removal from waste gases with a biological filter, Biotechnol. Bioeng. 25 (1983) 3089-3102.

[5] Z. Shareefdeen and B.C. Baltzis, Biofiltration of toluene vapor under steady-state and transient conditions: Theory and experimental results, Chem. Eng. Sci. 49 (1994) 4347-4360.

[6] S. M Zarook, A. A. Shaikh, Z. Ansar, Development, experimental validation and dynamic analysis of a general transient biofilter model, Chem. Eng. Sci. 52 (1997) 759-773. 
[7] J.S. Devinny, M. A. Deshusses, T.S. Webster, Biofiltration for air pollution control, Lewis publishers: Boca Raton, Florida, 1999.

[8] J.P. Arcangeli, E. Arvin, Modelling of toluene biodegradation and biofilm growth in a fixed biofilm reactor, Water Sci. Technol. 26 (1992) 617-626.

[9] C. Alonso, M.T. Suidan, G.A. Sorial, F. L. Smith, P. Biswas, P.J. Smith, R. C. Brenner, Gas treatment in trickle-bed biofilters: Biomass, how much is enough?, Biotechnol. Bioeng. 54 (1997) 583-594.

[10] I. Iliuta, F. Larachi, Transient biofilter aerodynamics and clogging for VOC degradation, Chem. Eng.Sci. 59 (2004) 3293-3302.

[11] A. Vergara-Fernandez, S. Hernandez, S. Revah, Phenomenological model of fungal biofilters for the abatement of hydrophobic VOCs, Biotechnol. Bioeng. 101 (2008) 11821192.

[12] F.J. Alvarez-Hornos, C. Gabaldon, V. Martinez-Soria, P. Marzal, J.M. Penya-Roja, Mathematical modeling of the biofiltration of ethyl acetate and toluene and their mixture, Biochem. Eng. J. 43 (2009) 169-177.

[13] G. Baquerizo, J.P. Maestre, T. Sakuma, M.A. Deshusses, X. Gamisans, D. Gabriel, J. Lafuente, A detailed model of a biofilter for ammonia removal: Model parameters analysis and model validation, Chem. Eng. J. 113 (2005) 205-214.

[14] A.D. Dorado, F. J. Lafuente, D. Gabriel, X. Gamisans, Biomass accumulation in a biofilter treating toluene at high loads. Part 1: Experimental performance from inoculation to clogging. Chem. Eng. J. (submitted)

[15] A.D. Dorado, G. Baquerizo, J.P. Maestre, X. Gamisans, D. Gabriel, J. Lafuente, Modeling of a bacterial and fungal biofilter applied to toluene abatement: Kinetic parameters estimation and model validation, Chem. Eng. J. 140 (2008) 52-61.

[16] A. Hoffmann, J.F. Mackowiak, A. Górak, M. Haas, J.M. Löning, T. Runowski, K. Hallenberger, Standardization of mass transfer measurements - A basis for the description of absorption processes, Chem. Eng. Res. Des. 85 (2007) 40-49.

[17] A.D. Dorado, F.J. Lafuente, D. Gabriel, X. Gamisans, The role of water in the performance of biofilters: parameterization of pressure drop and sorption capacities for common packing materials, J. Hazard. Mater. 180 (2010) 693-702.

[18]D.S. Hodge and J.S. Devinny, Modeling removal of air contaminants by biofiltration, J. Environ. Eng. 121 (1995) 21-32.

[19] M. Zilli, A. Del Borghi, A. Converti, Toluene vapour removal in a laboratory-scale biofilter, Appl. Microbiol. Biotechnol. 54 (2000) 248-254. 
[20] P.B. Leege, and W.H. Thompson, Test methods for the examination of composting and compost, US Composting Council: Bethesda, Vol. 1, 1995.

[21] A.D. Dorado, F.J. Lafuente, D. Gabriel, X. Gamisans, A comparative study based on physical characteristics of suitable packing materials in biofiltration, Environ. Eng. 31 (2010) 193-204.

[22]W. Mendenhall, and T. Sincich, Probabilidad y estadística para ingeniería y ciencias. Prentice Hall: México, Vol. 1, 1997. 


\section{TABLE CAPTIONS}

Table 1. Mathematical equations, boundary and initial conditions for gas phase (Eq. 1-3), biofilm phase (Eq. 4) and kinetic expressions (Eq. 5-10).

Table 2. Physical chemical and biological parameters used in the model.

\section{FIGURE CAPTIONS}

Figure 1. Experimental toluene profiles concentrations and model predictions at 3 different time operation in the calibration procedure of the model.

Figure 2. Experimental toluene concentrations in the 4 sampling ports along the time operation and model predictions in the calibration step.

Figure 3. Experimental evolution of parameters related with biomass growth and model predictions during the calibration period: pressure drop (A), porosity (B) and biomass weight (C).

Figure 4. Experimental toluene concentrations at the 4 sampling ports and model predictions in the validation step.

Figure 5. Experimental pressure drop and model predictions in the validation step.

Figure 6. Evolution of biofilm thickness predicted by the model in the 4 sampling ports (A) and biofilm thickness profiles along the height of the biofilter at 4 different time operations (B). 